\title{
BMJ Open FOOTPRINTS study protocol: rationale and methodology of a 3-year longitudinal observational study to phenotype patients with COPD
}

\author{
James Crapo (D) , ${ }^{1}$ Abhya Gupta, ${ }^{2}$ David A Lynch, ${ }^{3}$ Jens Vogel-Claussen, ${ }^{4,5}$ \\ Henrik Watz, ${ }^{6,7}$ Alice M Turner, ${ }^{8}$ Robert M Mroz, ${ }^{9}$ Wim Janssens, ${ }^{10}$ \\ Andrea Ludwig-Sengpiel, ${ }^{11}$ Markus Beck, ${ }^{12}$ Bérengère Langellier, ${ }^{13}$ Carina Ittrich, ${ }^{14}$ \\ Frank Risse, ${ }^{15}$ Claudia Diefenbach ${ }^{15}$
}

To cite: Crapo J, Gupta A, Lynch DA, et al. FOOTPRINTS study protocol: rationale and methodology of a 3-year longitudinal observational study to phenotype patients with COPD. BMJ Open 2021;11:e042526. doi:10.1136/ bmjopen-2020-042526

- Prepublication history and additional material for this paper are available online. To view these files, please visit the journal online (http://dx.doi. org/10.1136/bmjopen-2020042526).

Received 09 July 2020

Revised 01 December 2020

Accepted 18 February 2021

Check for updates

(C) Author(s) (or their employer(s)) 2021. Re-use permitted under CC BY-NC. No commercial re-use. See rights and permissions. Published by BMJ.

For numbered affiliations see end of article.

Correspondence to Professor James Crapo; crapoj@njhealth.org

\section{ABSTRACT}

Introduction A better understanding is needed of the different phenotypes that exist for patients with chronic obstructive pulmonary disease (COPD), their relationship with the pathogenesis of COPD and how they may affect disease progression. Biomarkers, including those associated with emphysema, may assist in characterising patients and in predicting and monitoring the course of disease. The FO0TPRINTS study (study 352.2069) aims to identify biomarkers associated with emphysema, over a 3-year period.

Methods and analysis The F00TPRINTS study is a prospective, longitudinal, multinational (12 countries), multicentre (51 sites) biomarker study, which has enrolled a total of 463 ex-smokers, including subjects without airflow limitation (as defined by the 2015 Global Initiative for Chronic Obstructive Lung Disease (GOLD) strategy report), patients with COPD across the GOLD stages 1-3 and patients with COPD and alpha1-antitrypsin deficiency. The study has an observational period lasting 156 weeks that includes seven site visits and additional phone interviews. Biomarkers in blood and sputum, imaging data (CT and magnetic resonance), clinical parameters, medical events of special interest and safety are being assessed at regular visits. Disease progression based on biomarker values and COPD phenotypes are being assessed using multivariate statistical prediction models.

Ethics and dissemination The study protocol was approved by the authorities and ethics committees/ institutional review boards of the respective institutions where applicable, which included study sites in Belgium, Canada, Denmark, Finland, Germany, Japan, Korea, Poland, Spain, Sweden, UK and USA; written informed consent has been obtained from all study participants. Ethics committee approval was obtained for all participating sites prior to enrolment of the study participants. The study results will be reported in peer-reviewed publications. Trial registration number NCT02719184.

\section{INTRODUCTION}

Airflow limitation is a key characteristic of chronic obstructive pulmonary disease (COPD). ${ }^{12}$ It is caused by small airways

\section{Strengths and limitations of this study}

- The study helps to address an unmet need to understand different chronic obstructive pulmonary disease (COPD) phenotypes and to expand on our understanding of biomarkers that are associated with emphysema, and hence COPD, in a 3-year longitudinal setting.

- A subset of patients with alpha1-antitrypsin deficiency provides information on an important but rarely studied subpopulation of patients that present with earlier onset and faster progression of emphysema.

- A regular sampling schedule is employed, with frequent visits and biomarker sample collections to allow early detection of changes, if present.

- MRI and sputum collection only occur at certain study sites and not necessarily at all planned times; hence, the smaller subsets of patients may not be representative of the entire population.

- Patients with the newly defined preserved ratio impaired spirometry phenotype are not included, nor are current smokers or healthy subjects who have never smoked, thus limiting the control groups for reference.

disease, such as obstructive bronchiolitis, and structural changes related to parenchymal destruction (emphysema). ${ }^{2}$ Small airways disease and structural changes can occur alone or in combination with one another, with the degree of severity and relative contributions varying between individuals. ${ }^{1}$

COPD is associated with inflammation and tissue damage, which is thought to be linked to-among other aspects-an imbalance between neutrophil serine proteases (NSPs) and their inhibitors. ${ }^{3} 4$ The serine protease inhibitor alpha1-antitrypsin (A1AT) inactivates NSPs such as neutrophil elastase (NE), proteinase 3 (PR3) and cathepsin G 
(Cat $G$ ) to protect lung tissue against protease-mediated damage. ${ }^{56}$ Although a number of gene defects have been associated with emphysema, ${ }^{7}$ A1AT deficiency (A1ATD) is the most well established as a cause for COPD. ${ }^{8}$ It is associated with a higher risk of developing emphysema that tends to occur at a younger age and progresses faster than for patients without A1ATD. ${ }^{510}$ Subjects with the most severe A1ATD are usually homozygous for the ZZ allele. ${ }^{5}$ The resulting emphysema among patients with A1ATD is thought to be caused primarily by increased NSP activity in the lungs. ${ }^{10}$

NE and PR3 are responsible for the degradation of critical components of the extracellular matrix, including elastin and fibronectin, and can produce other key features of COPD, such as mucous hypersecretion. ${ }^{3}$ When quantified, levels of NE, PR3 or their specific elastin degradation products have been associated with poorer disease outcomes in patients with COPD, such as incidence of exacerbations and higher risk of mortality. ${ }^{11-13}$ Other proteases and biomarkers, including desmosine, fibrinogen and $\mathrm{C}$ reactive protein, have also been associated with poorer disease outcomes in patients with COPD. ${ }^{13-15}$

In the era of precision-based patient care, there is a need for a more in-depth understanding of COPD patient phenotypes, pathogenesis and progression. ${ }^{16} 17$ Biomarkers may assist in characterising patients and in predicting and monitoring the course of disease, in order to better enable the development of drugs aimed at slowing COPD progression. ${ }^{16-18}$ In fact, there are encouraging signs that therapies that modify NSP imbalance (eg, augmentation therapy for A1ATD) appear to alter emphysema progression, at least when considering blood and radiological biomarkers of disease ${ }^{19}{ }^{20}$ Since quantitative CT is a well-established method for documenting the presence, extent and progression of emphysema, it is potentially useful for assessing these emerging biomarkers. ${ }^{21-24}$

This prospective, longitudinal study is investigating biomarkers in different biofluids (whole blood, serum, plasma and induced sputum), imaging parameters assessed by CT and MRI, as well as clinical parameters potentially associated with emphysema, over a 3-year period. It is hoped that correlation of lung destruction biomarkers with CT densitometry findings should increase our understanding of the underlying pathophysiology of emphysema progression. The insights generated may support the development of future treatments that could slow or halt disease progression in patients with COPD. Here we report the methodology of the FOOTPRINTS study, as described in the study protocol (V.5.0; date: 29 May 2018).

\section{Objectives}

The primary objective of this longitudinal study is to explore whether any of the biomarkers assessed in patients with COPD, patients with COPD plus A1ATD and subjects without airflow limitation (controls; as defined by the 2015 Global Initiative for Chronic Obstructive Lung Disease (GOLD) strategy report), ${ }^{25}$ or specific patterns thereof, are correlated with COPD progression, particularly emphysema progression, over 156 weeks.

Further objectives, to be assessed over 156 weeks, include:

- To assess the correlation of soluble biomarkers of inflammation and lung tissue destruction with COPD severity, presence of emphysema and disease progression.

- To determine the concentration and activity of proteases in induced sputum and to assess potential biomarkers of lung tissue destruction in sputum and blood, from patients with different severities of COPD (including a subset of patients with A1ATD) and subjects without airflow limitation (controls).

- To assess the correlation of physiological biomarkers and functional imaging biomarkers with COPD severity, the presence of emphysema and disease progression and to evaluate MRI-based imaging biomarkers with respect to their feasibility.

- To identify COPD phenotypes and define their risk for, and rate of, disease progression.

\section{METHODS AND ANALYSIS \\ Study design}

The FOOTPRINTS study (registered on 21 March 2016) is a multinational, prospective, longitudinal, biomarker study in subjects without airflow limitation, patients with COPD and patients with COPD and A1ATD, taking place at 51 sites across 12 countries (Belgium, Canada, Denmark, Finland, Germany, Japan, Korea, Poland, Spain, Sweden, UK and USA). A complete list of study sites is detailed in online supplemental table 1.

The study consists of two consecutive periods (a screening period lasting up to 28 days and an observational period lasting 156 weeks) and includes seven site visits and additional phone interviews (figure 1). The observational period is completed at visit 7 or at the discontinuation visit, which is regarded as study completion. Patients are considered to be in the observational phase only after completing visit 2. All subjects who prematurely discontinue the study during the observational phase are followed for vital status and COPD exacerbation status (for patients with COPD and for patients with COPD and A1ATD only) via phone contact until 156 weeks after visit 2 .

Calculation of sample size and power calculations for annual decline in forced expiratory volume in $1 \mathrm{~s}\left(\mathrm{FEV}_{1}\right)$ and 15th percentile density assessed by CT are provided in online supplemental text 1 and tables 2 and 3 .

\section{Inclusion and exclusion criteria}

Male and female participants who have signed an informed consent form consistent with the International Conference on Harmonisation Good Clinical Practice (GCP) guidelines prior to participation have been included, provided the following were applicable:

- Aged 40-70 years (30-70 years for patients with COPD and A1ATD). 


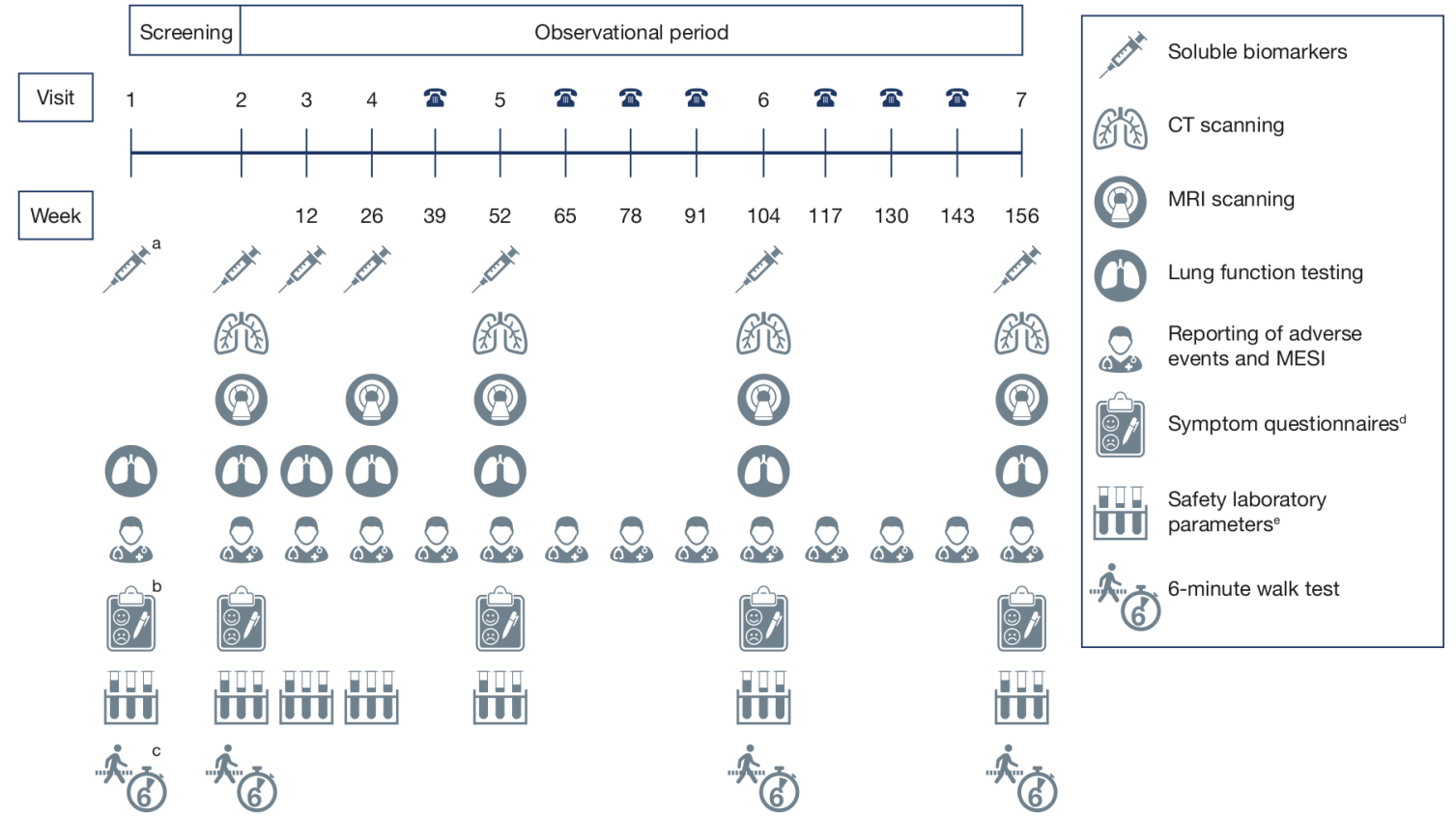

Figure 1 Overview of trial design ${ }^{\mathrm{a}} \mathrm{A} 1 \mathrm{ATD}$ analysis only; this occurs at visit 1 only. ${ }^{\mathrm{b}} \mathrm{mMRC}$ only. ${ }^{\mathrm{c}} \mathrm{At}$ visit 1 , the 6 -min walk test is being performed to train the patients for the procedure. ${ }^{d}$ Symptom questionnaires include the CAT, mMRC and SGRQ. The CAT, mMRC and SGRQ are being conducted in patients with COPD and with COPD plus A1ATD only. ${ }^{\mathrm{e} A}$ full panel of haematology, blood chemistry and coagulation parameters is performed at visits 1,2, 5, 6 and 7, with a reduced panel comprising haematology, differential automatic cell counts, fibrinogen, highly sensitive $\mathrm{C}$ reactive protein and creatinine performed at visits 3 and 4. A1ATD, alpha1-antitrypsin deficiency; CAT, COPD assessment test; COPD, chronic obstructive pulmonary disease; MESI, medical events of special interest; mMRC, modified Medical Research Council dyspnoea scale; SGRQ, St. George's Respiratory Questionnaire.

- Ex-smokers for $\geq 9$ months with a smoking history of $\geq 20$ pack-years ( $\geq 10$ pack-years for patients with COPD and A1ATD).

- Body mass index $18-35 \mathrm{~kg} / \mathrm{m}^{2}\left(\leq 30 \mathrm{~kg} / \mathrm{m}^{2}\right.$ in the MRI subset).

- Able to perform all study-related procedures.

- Required to have been on stable therapy for 4 weeks prior to visit 1.

Participants were excluded if any of the following were applicable:

- Significant pulmonary disease (other than COPD) or other significant medical condition, for example, rheumatoid arthritis, severe liver disease or psoriasis.

- Documented history of asthma, including during childhood.

- Any respiratory tract infection or COPD exacerbation within 4 weeks prior to visit 1 or during the screening period prior to visit 2, if it was not possible to meet the rescheduling rules.

- Presence of an immunocompromising condition (eg, HIV or history of hepatitis B or C).

- Any treatment with phosphodiesterase-4 inhibitors and maintenance treatment with methylxanthines within 3 months or 6 half-lives prior to visit 1 and until visit 2; patients were permitted to initiate treatment following visit 2.

- Patients with COPD (with and without A1ATD) with newly added anti-inflammatory treatment (either respiratory or non-respiratory) or change in any therapy within 4 weeks prior to visit 1 and during the screening period between visit 1 and visit 2; patients were permitted to initiate treatment following visit 2.

- Any prior, current or planned A1AT augmentation therapy.

- Permitted and restricted medications and therapy are detailed in online supplemental table 4.

- Pregnant or lactating women.

- Previous participation in this study or participation in a parallel interventional clinical trial within 6 weeks prior to visit 1 or during the study.

\section{Patient population and recruitment}

The study is ongoing; however, enrolment is complete. In total, 463 patients were enrolled, who are described in more detail in table 1 . Strategies for achieving adequate participant enrolment and retainment are described in online supplemental text 1 .

The study started on 22 July 2016 when the first subject was screened, and the planned end date for the study is 29 March 2021.

\section{Main outcome measures}

The main outcome measures are change in lung density (assessed by CT), lung function decline, and number, duration and severity of exacerbations over the course of the study. 
Table 1 FOOTPRINTS study participants: all enrolled subjects

\begin{tabular}{|c|c|}
\hline Patient group & Rationale \\
\hline $\begin{array}{l}383 \text { patients with COPD, including: } \\
123 \text { patients with mild COPD* } \\
130 \text { patients with moderate COPD } 1 \\
130 \text { patients with severe COPD } \$\end{array}$ & $\begin{array}{l}\text { Included to provide data on increased lung protease levels and the } \\
\text { contribution that this may have on the development and progression of } \\
\text { emphysema in the absence of A1ATD. }\end{array}$ \\
\hline 18 patients with COPD and A1ATD§ & $\begin{array}{l}\text { Included as they present with an earlier onset of emphysema and a faster } \\
\text { decline in lung function, as well as a faster change in lung density. }{ }^{9}\end{array}$ \\
\hline 62 ex-smokers without airflow limitation & 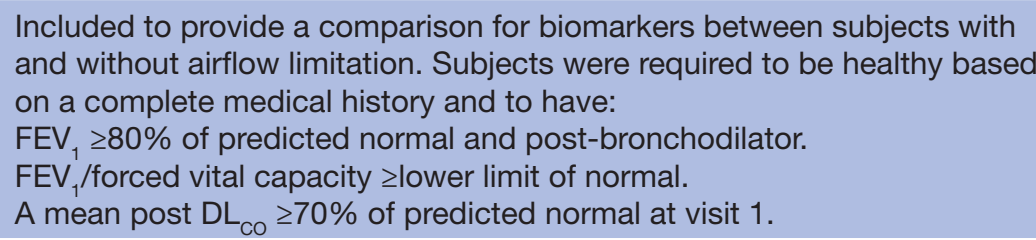 \\
\hline
\end{tabular}

\section{Total enrolled: 463 subjects}

\section{*Defined as GOLD stage $1, \mathrm{FEV}_{1} \geq 80 \%$ predicted. $^{25}$}

†Defined as GOLD stage 2, FEV $1 \geq 50-<80 \%$ predicted. ${ }^{25}$

$\neq$ Defined as GOLD stage 3, FEV $1 \geq 30-<50 \%$ predicted. $^{25}$

$\S$ Ddefined as having a diagnosis of COPD and a documented A1ATD of ZZ genotype prior to visit 2.

A1ATD, alpha1-antitrypsin deficiency; COPD, chronic obstructive pulmonary disease; $\mathrm{DL}_{\mathrm{CO}}$, diffusing capacity of the lungs for carbon

monoxide; $F_{1}$, forced expiratory volume in $1 \mathrm{~s}$; GOLD, Global Initiative for Chronic Obstructive Lung Disease; ZZ, ZZ allele.

\section{Planned analyses and assessments}

Variables to be analysed include a range of soluble biomarkers, imaging biomarkers and clinical parameters, as well as medical events of special interest (MESI) and safety assessments. The pre-specified biomarkers being assessed are summarised in table 2, and the biomarker sampling schedule and overall trial design are illustrated in figure 1.

Blood and sputum are being collected at visits $2-7$ for analysis of pre-specified soluble biomarkers, and blood is also being collected at visit 1 to perform a genetic test for A1ATD (figure 1). Total and differential cell counts in blood, which are being assessed within the safety laboratory assessments, are also being assessed as biomarkers. Sputum will be induced to generate sputum samples, including cell slides to assess total and differential cell count and supernatant to assess soluble biomarker levels (table 2).

Chest CT scans are being conducted annually at visits 2, 5, 6 and 7, following a specific low-dose protocol in order to minimise radiation exposure at each visit. A COPDGene phantom ${ }^{26}$ is being used before the first patient scan and then bi-monthly to monitor the stability of CT measurements for each scanner. MRI evaluation of the pulmonary vasculature and right ventricle is conducted in a subset of patients without airflow limitation and patients with COPD (not in patients with COPD and A1ATD) at visits 2, 4, 5, 6 and 7. MESI include respiratory disorders such as pneumonia, pulmonary fibrosis, bronchiectasis and COPD exacerbations, as well as any other disorders (eg, cardiovascular, metabolic/digestive and neurological/psychiatric) reported at the investigator's discretion. COPD exacerbations are defined as new or increased lower respiratory events or symptoms related to the underlying COPD with duration $\geq 3$ days. Assessment of safety is carried out at visits 1, 5, 6 and 7 unless otherwise indicated, including physical examination (plus assessment of height (visit 1 only) and weight), vital signs (blood pressure and pulse rate), safety laboratory parameters (from blood; assessed at every site visit (visits 1-7)) and ECG. Adverse events associated with any study procedure and MESI are collected at every site visit (visits 1-7; MESI also assessed via telephone contact between visits; figure 1). All adverse events are followed up until they have resolved, been sufficiently characterised or no further information can be obtained.

\section{Rescheduling of clinic visits}

Any deviations from the planned visit schedule are being documented. Rescheduling of certain clinic visits was permitted to promote participant retention. Clinic visits that involve a lung function test may be rescheduled twice within the permitted time windows due to violation of lifestyle restrictions. Subjects should refrain from strenuous activity for at least 12 hours prior to lung function testing (and throughout the testing period) and should avoid cold temperatures, environmental smoke, dust or areas with strong odours (eg, perfumes). Sputum induction at visit 2 can be rescheduled by up to 3 days; at all subsequent visits, it should not be repeated if the subject is unable to produce sputum or if the sputum sample quality is not acceptable. If chest CT and MRI (where applicable) cannot be performed on the day of the scheduled visit, the imaging assessment can be rescheduled up to 14 days after the visit. If a patient experiences a COPD exacerbation or respiratory tract infection during the screening period, visit 2 will be postponed until 4 weeks following recovery from the exacerbation or infection, and the screening period may be extended up to 8 weeks. 
Table 2 Summary of biomarker assessments

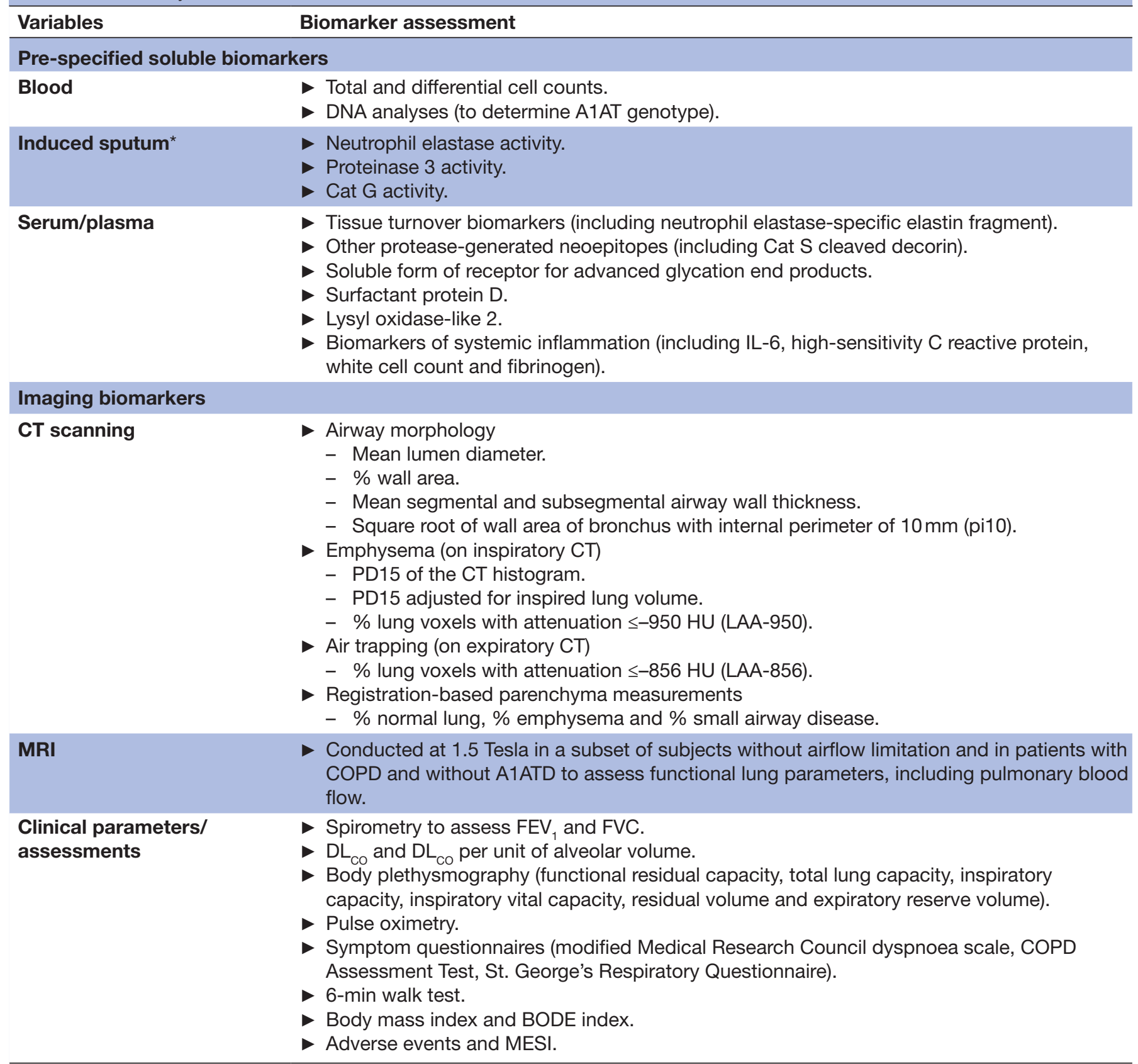

${ }^{*}$ Collected at selected sites.

A1AT, alpha1-antitrypsin; A1ATD, alpha1-antitrypsin deficiency; BODE, Body mass index, airway Obstruction, Dyspnea, and Exercise; Cat, cathepsin; COPD, chronic obstructive pulmonary disease; $\mathrm{DL}_{\mathrm{cO}}$, diffusing capacity of the lungs for carbon monoxide; FEV $\mathrm{V}_{1}$, forced expiratory volume in 1 s; FVC, forced vital capacity; HU, Hounsfield Units; IL-6, interleukin-6; LAA, low attenuation areas; MESI, medical events of special interest; PD15, lung density at the 15th percentile point of the CT histogram.

\section{Statistical analysis}

Biomarker assessments and clinical outcomes measures are evaluated by regression models for repeated measures using respective point estimates and confidence interval estimates. The correlation between individual biomarkers, and between biomarkers and other clinical outcome measures, is being assessed. Multivariate statistical prediction models for disease progression based on biomarker values and COPD phenotypes are being developed and examined. Interim analyses will also be conducted by the trial team once data from the 52-week and 104-week assessments are available for $\geq 70 \%$ of subjects entered into the study. The purpose of this is to provide information on the use of MRI and soluble biomarker assessments.

\section{Information on steering committee}

A scientific steering committee, Emphysema Progression Biomarker Study (FOOTPRINTS study) Steering Committee, was established in January 2017 to discuss and evaluate key scientific data in the field of respiratory 
medicine and to provide independent advice and recommendations to optimise and strengthen the FOOTPRINTS biomarker study. The steering committee comprises 13 external and internal scientific experts in respiratory medicine and chest radiology (online supplemental table 5 ) and is conducted in adherence to industry regulations on the cooperation of the pharmaceutical industry with the medical professions. ${ }^{27-29}$

\section{Data collection and management}

An interactive response technology system was used to track subject enrolment and to register clinic visits and phone calls. A central laboratory facility is handling all standard safety blood laboratory analyses. Spirometry is performed using an external vendor's equipment to allow for centralised readings. ECGs are recorded for about a 10-s duration after the subject has rested for at least $5 \mathrm{~min}$ in a supine position, with electrode placement performed according to the Wilson method (precordial leads) and Goldberger and Einthoven (limb leads). Pulse oximetry will be performed to measure oxygen saturation; all recordings will be made using the same pulse oximetry measurement device per site on the same finger of the subject. Body plethysmography, diffusing capacity of the lungs for carbon monoxide $\left(\mathrm{DL}_{\mathrm{CO}}\right)$, the 6-min walk test (6MWT) and sputum induction and processing (at selected sites only) are performed according to international accepted guidelines as follows. Body plethysmography and $\mathrm{DL}_{\mathrm{CO}}$ measurement are being performed according to American Thoracic Society (ATS)/ European Respiratory Society guidelines, after salbutamol administration. ${ }^{30}$ The 6MWT is being conducted according to ATS guidelines. ${ }^{31}$ Inspiratory and expiratory CT scans are being performed according to the specifications provided in online supplemental table 6. Sputum induction and processing are performed according to the sputum manual issued by the vendor for sputum assessment and analysis.

All samples collected for pre-specified biomarker assessments are prepared by the site according to instructions given in the investigator site file until shipment to the central contract research organisation. Additional blood samples (whole blood, plasma and serum) will be taken at visits $2-7$ for future unspecified analyses, while potential leftovers from pre-specified blood analysis and induced sputum will also be stored and used for this purpose; these samples will be stored for up to 15 years after the final study report has been signed. Biomarkers are analysed by the sponsor or contractors of the sponsor. Data management and statistical evaluation are conducted by the sponsor or contractors of the sponsor. Source documents are filed at the investigator's site; these data are transferred to the electronic case report form. Missing data may be imputed if necessary.

A clinical trial monitor, appointed by the sponsor, ensures the good running of the study and directs the clinical study team in the preparation, conduct and reporting of the study, in accordance with the sponsor's standardised operating procedures, GCP guidelines and current legislation.

Medical information obtained for individual subjects during the study is considered confidential, and this will be ensured by using subject identification code numbers. Further information on confidentiality is detailed in the informed consent form (online supplemental file 2).

\section{Patient and public involvement}

Patients or the public were not involved in the design, conduct, reporting or dissemination plans of our research.

\section{ETHICS AND DISSEMINATION}

This study is being performed in accordance with the International Conference on Harmonisation Tripartite Guideline for GCP, the Japanese GCP regulations and local regulations. The study protocol has received approvals from the following institutional ethics committees (IECs)/institutional review boards (IRBs): Belgium: Ethische commissie onderzoek UZ/KU Leuven; Canada: Hamilton Integrated Research Ethics Board, Ontario; Research Ethics Board University of Cardiology and Pneumology Institute of Quebec; Health Research Ethics Board, University of Alberta; University of Saskatchewan Biomedical Research Ethics Board; Denmark: De Videnskabsetiske Komitéer for Region Hovedstaden, Hillerød; Finland: Varsinais-Suomen sairaanhoitopiiri Eettinen toimikunta; Germany: Ethikkommission Landesärztekammer Hessen, Frankfurt; Ethikkommission Schleswig-Holstein, Bad Segeberg; Ethikkommission der Medizinischen Hochschule Hannover, Hannover; Japan: the IRB of Osaka City University Hospital, Osaka; the IRB of Kishiwada City Hospital, Kishiwada; the IRB of Showa University Hospital, Shinagawa; the IRB of Kagoshima University Hospital, Kagoshima; Republic of Korea: The Catholic University of Korea, Eunpyeong St. Mary's Hospital IRB, Seoul; Seoul Metropolitan Government Seoul National University Boramae Medical; Korea University Guro Hospital IRB, Seoul; Konkuk University Medical Center IRB, Seoul; Poland: Bioethics Committee at Regional Medical Chamber, Bialystok; Spain: CEIm Hospital Clínic, Barcelona; CEIC Illes Balears, Palma; CEIC Hospital Universitari Vall d'Hebrón, Barcelona; CEICParc de Salut Mar, Barcelona; CEIC - Hospital de Bellvitge, Barcelona; Sweden: Regionala etikprövningsnämnden i Lund, Lund; UK: North West - Greater Manchester East Research Ethics Committee; USA: University of Alabama at Birmingham Western Institutional Review Board, Puyallup; Baylor College of Medicine Institutional Review Board for Baylor, Houston; University of California San Diego UCSD Human Research Protection Program, La Jolla; Temple University Hospital Western Institutional Review Board, Puyallup; University of Utah Health Sciences Center University of Utah IRB, Salt Lake City; University of California Los Angeles Western Institutional Review Board, Puyallup; Chesapeake IRB, Columbia; Advarra, Inc, Columbia; University of Iowa Human 
Subjects Office/Institutional Review Board, Iowa City; Johns Hopkins Institutional Review Board Reed Hall - B 130, Baltimore; National Jewish Health Western Institutional Review Board, Puyallup.

In the respective countries, the ethics approval preceded the study participant's enrolment. Written informed consent is obtained from all study participants. Notably, any protocol amendments will be initiated only after all required legal documentation has been reviewed and/or approved by the respective IRB/IEC, as applicable.

The study findings will be reported in due course at research conferences and in peer-reviewed journals. The study protocol and clinical study data will be available to be shared after publication of the primary manuscript in a peer-reviewed journal. These data will be available on reasonable request; further details will be provided in the primary manuscript.

\section{DISCUSSION}

COPD is characterised by progressive airflow limitation and a decline in lung function. It has also been demonstrated that emphysema, a major cause of airflow limitation in COPD, is independently associated with a rapid decline in lung function. ${ }^{32} 33$ Current pharmacological treatment options for COPD and emphysema do not prevent the progressive decline of $\mathrm{FEV}_{1}$ - the most commonly used marker of disease severity and progression in COPD. ${ }^{134} 35$ However, $\mathrm{FEV}_{1}$ is poorly correlated with symptoms and some other measures of disease progression. ${ }^{136}$

The heterogeneity of COPD extends to a molecular level, a concept familiar in asthma, where endotype-directed therapy is now well accepted; ${ }^{37}$ hence, there is a need for biomarkers that can assist with diagnosis, risk stratification and assessment of therapeutic interventions. ${ }^{38} 39 \mathrm{~A}$ recent systematic review by Fermont $e t a l^{14}$ reported that biomarkers and clinical parameters including 6MWT, fibrinogen, $\mathrm{C}$ reactive protein, white cell count and interleukin-6, are associated with clinical outcomes in COPD and worth further investigation. A systematic review of CT density as a radiological biomarker came to similar conclusions. ${ }^{40}$ It is anticipated that biomarkers may facilitate a better understanding of the prognosis of COPD, help to guide treatment options and allow for a greater degree of precision medicine as treatment options. ${ }^{38}$

So far, few studies have addressed the potential of biomarkers to predict the longitudinal outcomes of COPD, and this has been assessed for a limited number of biomarkers only. The COPDGene study, which has enrolled over 10000 smoking (with or without COPD) and non-smoking subjects, is predominantly focused on identifying genetic determinants for COPD susceptibility, ${ }^{41}$ while the UK-based British Lung Foundation early COPD study is currently enrolling smokers with normal lung function or mild lung function abnormalities to assess the very early stages of COPD development. ${ }^{42}$ The Evaluation of COPD Longitudinally to Identify Predictive
Surrogate End-points (ECLIPSE) study ${ }^{43}$ and the Subpopulations and Intermediate Outcomes in COPD Study

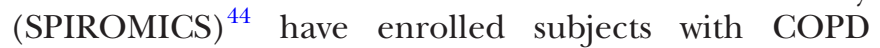
and smoking/non-smoking control subjects, similar to the COPDGene study. Other longitudinal studies include the German COPD and SYstemic consequencesCOmorbidities NETwork (COSYCONET) study ${ }^{45}$ and the Canadian Cohort Obstructive Lung Disease (CanCOLD) study. ${ }^{46}$ All of these studies plan to analyse a range of soluble biomarkers and clinical outcomes and conduct CT imaging to help identify COPD phenotypes. ${ }^{42-48}$

This prospective, longitudinal study was designed to investigate, over a 3-year period, biomarkers in different biofluids, imaging biomarkers and clinical parameters that may be associated with emphysema. A key strength of the FOOTPRINTS study is that it actively recruited a subset of patients with A1ATD. This is an important but rarely studied subpopulation of patients, which has not been actively recruited for in other longitudinal studies, such as the COPDGene, ${ }^{41}$ ECLIPSE, ${ }^{43}$ SPIROMICS ${ }^{44}$ and UK early COPD cohort studies. ${ }^{48}$ Patients with A1ATD present with earlier onset of emphysema and could help us to better understand possible markers for emphysema progression, in particular the role of NSP imbalance conditional on A1AT levels. ${ }^{10}$ While A1ATD is associated with a higher risk of emphysema and COPD, increased levels of serum A1AT occur in response to inflammation. ${ }^{49}$ Interestingly, increased levels of A1AT have also been associated with worse outcomes in patients with COPD. A recent analysis of data from the Hokkaido COPD cohort study examined the association of circulating A1AT levels with the clinical course of COPD patients without A1ATD and reported that higher A1AT levels were associated with worse outcomes, including more emphysema, a worse systemic inflammation status and higher 10-year mortality. ${ }^{49}$ Our findings in patients with A1ATD could help us to further understand the complex relationship between A1AT levels and COPD disease progression.

Compared with the COPDGene study, in which assessments were scheduled at baseline, 5 years and 10 years (ongoing), ${ }^{414} 470$ the FOOTPRINTS study has more frequent visits and biomarker sample collections, similar to the ECLIPSE ${ }^{43}$ and SPIROMICS ${ }^{44}$ studies. In particular, the regular collection of sputum is a major asset of the FOOTPRINTS study; sputum collection is scheduled to occur more frequently than in both the COPDGene ${ }^{47}$ and SPIROMICS ${ }^{44}$ studies and with a similar frequency to the ECLIPSE ${ }^{43}$ study, with four collections scheduled in the first year of the study and subsequent collections scheduled at year 2 and year 3 . Another key strength and differentiator of the FOOTPRINTS study is that annual chest CT scans are being performed; compared with the other longitudinal studies discussed here, and to the best of our knowledge, the FOOTPRINTS study will provide the most regular CT imaging data. This, in addition to the frequent biomarker sampling, will allow for more robust measures of short-to-medium-term decline, critical for informing clinical trials of emphysema-targeted 
medicinal products. ${ }^{434}$ The FOOTPRINTS study is also assessing a wider range of non-genetic biomarkers and clinical parameters across the entire study population compared with the COPDGene study, which is focused primarily on genetic analyses of DNA samples. ${ }^{414}$ For example, the FOOTPRINTS study is assessing NE, PR3 and Cat $G$ activity, which could help us to further understand the role of these biomarkers in patients with COPD, including those with A1ATD and poorer disease prognosis. ${ }^{10}$ In addition, FOOTPRINTS is conducting MRI assessments, which could be valuable in improving our understanding of changes in vasculature and perfusion in the lung that may precede changes in emphysema. ${ }^{51}$ Due to the small number of patients included in the subset analysis and the challenges associated with obtaining meaningful MRI data, these findings are expected to be limited.

Although the FOOTPRINTS study is not focusing on frequent exacerbators, it is anticipated that a considerable number of adverse events, MESI and hospital admissions will be detected during the 3 years of follow-up. The wide range of biomarkers measured in sputum and blood may help identify subjects at risk for such events. In addition, the longitudinal biosampling in FOOTPRINTS will offer a unique opportunity to study the impact of exacerbations on the evolution of pathogenic processes.

It is anticipated that the results of the FOOTPRINTS study will complement, as well as expand on, the data generated in other longitudinal studies, such as COPDGene, ${ }^{41}$ ECLIPSE $^{43}$ and SPIROMICS. ${ }^{44}$ It is envisaged that the results of this study will increase our understanding of COPD phenotypes and the underlying pathophysiology of emphysema progression, which may be of use when developing drugs to reduce COPD advancement. In addition, biomarkers associated with ongoing emphysematous destruction of lung parenchyma may be identified, which could assist in predicting and monitoring patients' COPD disease course.

A potential limitation of the FOOTPRINTS study is that sputum collection and MRI only occur at certain study sites. As such, the smaller subset of subjects may not be sufficiently large to represent the entire population. In addition, there is potential for selection bias at site and patient levels, as the study is non-randomised and all participants are required to be ex-smokers. Note that current smokers were not included because smoking cessation can cause high variability in outcomes, particularly lung density measurements. ${ }^{52}$ Given that disease progression occurs despite smoking cessation, ex-smokers were considered to be a more appropriate population. A further limitation is that patients with the newly defined preserved ratio impaired spirometry phenotype ${ }^{415455}$ and healthy subjects who have never smoked are not included.

Finally, an inherent challenge in studying biomarker levels in patients with COPD is the variability in biomarker levels over time. For example, a study by Dickens $e t a l^{39}$ looked at variability in levels of biomarkers over 3 months in subjects with COPD. At month 3, fibrinogen was a highly repeatable biomarker, with levels within $25 \%$ of their baseline values for $89 \%$ of study participants, yet only $21 \%$ of patients had levels of $\mathrm{C}$ reactive protein within $25 \%$ of baseline values. In the current study, the lack of a healthy never-smoker control group limits the opportunity to compare the extent of biomarker fluctuations over time. The reasons for variability over time are poorly understood, but the repeatability of biomarkers should be considered when selecting for clinical applications. ${ }^{39}$ Therefore, longitudinal assessment of biomarkers is planned to help understand the longitudinal stability of biomarker-based phenotypes.

In summary, biomarkers may help to characterise patients with COPD, allow for better monitoring and prediction of the disease course and enable an increased understanding of COPD itself. Subsequently, this should help us to develop drugs to reduce disease progression. The FOOTPRINTS longitudinal study is investigating biomarkers in biofluids, imaging biomarkers and clinical parameters associated with emphysema over a 3-year period to increase the understanding of COPD patient phenotypes, pathogenesis and disease progression.

\section{Author affiliations}

${ }^{1}$ Department of Medicine, National Jewish Health, Denver, Colorado, USA

${ }^{2} \mathrm{TA}$ Inflammation Med, Boehringer Ingelheim International GmbH, Biberach an der Riss, Germany

${ }^{3}$ Department of Radiology, National Jewish Health, Denver, Colorado, USA

${ }^{4}$ Department of Diagnostic and Interventional Radiology, Hannover Medical School, Hannover, Germany

${ }^{5}$ Biomedical research in endstage and obstructive lung disease Hannover (BREATH), German Center for Lung Research (DZL), Hannover, Germany

${ }^{6}$ Pulmonary Research Institute, LungenClinic Grosshansdorf, Grosshansdorf, Germany

${ }^{7}$ Airway Research Center North (ARCN), German Center for Lung Research (DZL), Grosshansdorf, Germany

${ }^{8}$ Institute of Applied Health Research, University of Birmingham, Birmingham, UK ${ }^{9}$ 2nd Department of Lung Diseases and Tuberculosis, Bialystok Medical University, Bialystok, Poland

${ }^{10}$ Department of Chronic Diseases, Metabolism and Ageing (CHROMETA), Laboratory of Respiratory Diseases and Thoracic surgery (BREATH), University Hospital Leuven, $\mathrm{KU}$ Leuven, Belgium

${ }^{11} \mathrm{KLB}$ Gesundheitsforschung Lübeck GmbH, Lübeck, Germany

${ }^{12}$ Department of Clinical Research, Boehringer Ingelheim Pharma GmbH \& Co. KG, Biberach an der Riss, Germany

${ }^{13}$ Department of Medical Affairs, Boehringer Ingelheim France SAS, Paris, France

${ }^{14} \mathrm{Global}$ Department of Biostatistics and Data Sciences, Boehringer Ingelheim Pharma GmbH \& Co. KG, Biberach an der Riss, Germany

${ }^{15}$ Department of Translational Medicine and Clinical Pharmacology, Boehringer Ingelheim Pharma GmbH \& Co. KG, Biberach an der Riss, Germany

Acknowledgements Vicki Cronin, PhD, at MediTech Media provided editorial assistance in the development of this manuscript, funded by Boehringer Ingelheim, and Harald Koegler, MD, assisted with reviewing the manuscript. The authors would like to thank all the patients and healthy volunteers for participating in this trial and also extend their thanks to the investigators and study staff.

Contributors JC, AG, DAL, JV-C, HW, MB, Cl, FR and CD contributed towards the overall concept and design of the original study protocol. AMT also contributed towards the study design by providing advice on the inclusion criteria for patients with alpha1-antitrypsin deficiency. BL, CD, MB and $\mathrm{Cl}$ led the writing of the protocol. JC, AG, DAL, JV-C, HW, MB, BL, Cl, FR and CD provided critical input at all stages. HW, AMT, RMM, WJ and AL-S are principal investigators of the study and were involved in enrolling patients, as well as documenting patient information in the trial database. All authors have contributed towards the development of the manuscript and have read and approved the final manuscript. 
Funding This study was funded and sponsored by Boehringer Ingelheim. The trial clinical monitor for this study was Markus Beck (Boehringer Ingelheim Pharma GmbH \& Co. KG, Department of Clinical Research Germany/Respiratory Diseases, Birkendorfer Str. 65, 88397 Biberach an der Riss, Germany; Phone: +49 (0) 7351/54 96 803)

Competing interests JC is a co-prinicipal investigator for the COPDGene study and has received grants from National Institutes of Health/National Heart, Lung, and Blood Institute (NHLBI). AG, MB, BL, Cl, FR and CD are employees of Boehringer Ingelheim. DAL has received grants from NHLBl, and personal fees from Boehringer Ingelheim, Parexel, Siemens and Veracyte. In addition, DAL has a patent for 'systems and methods for classifying severity of COPD' pending. HW has received grants and personal fees from Boehringer Ingelheim, and personal fees from AstraZeneca, Chiesi, GSK, Menarini and Novartis. JV-C has received grants and personal fees from Boehringer Ingelheim and Novartis, and grants from GSK and Siemens Healthineers. AMT has received personal fees, payment for educational talks and expenses payments for work on the FOOTPRINTS steering committee from Boehringer Ingelheim, grants and personal fees from AstraZeneca, CSL Behring and Grifols Biotheraputics, and grants, personal fees and non-finanacial support from Chiesi. RMM has received personal fees from AstraZeneca, Boehringer Ingelheim, GSK, Merck Sharp \& Dohme, Mundipharma, Novartis and Roche. WJ has received grants from AstraZeneca and Chiesi. AL-S reports receipt of a study fee for involvement in conducting the FO0TPRINTS study.

Patient consent for publication Not required.

Provenance and peer review Not commissioned; externally peer reviewed.

Supplemental material This content has been supplied by the author(s). It has not been vetted by BMJ Publishing Group Limited (BMJ) and may not have been peer-reviewed. Any opinions or recommendations discussed are solely those of the author(s) and are not endorsed by BMJ. BMJ disclaims all liability and responsibility arising from any reliance placed on the content. Where the content includes any translated material, BMJ does not warrant the accuracy and reliability of the translations (including but not limited to local regulations, clinical guidelines, terminology, drug names and drug dosages), and is not responsible for any error and/or omissions arising from translation and adaptation or otherwise.

Open access This is an open access article distributed in accordance with the Creative Commons Attribution Non Commercial (CC BY-NC 4.0) license, which permits others to distribute, remix, adapt, build upon this work non-commercially, and license their derivative works on different terms, provided the original work is properly cited, appropriate credit is given, any changes made indicated, and the use is non-commercial. See: http://creativecommons.org/licenses/by-nc/4.0/.

\section{ORCID iD}

James Crapo http://orcid.org/0000-0002-1262-5230

\section{REFERENCES}

1 Global Initiative for Chronic Obstructive Lung Disease. Global strategy for the diagnosis, management, and prevention of chronic obstructive pulmonary disease (2020 report), 2019. Available: https:// goldcopd.org/wp-content/uploads/2019/11/GOLD-2020-REPORTver1.0wms.pdf [Accessed 15 Jun 2020].

2 Bourdin A, Burgel P-R, Chanez P, et al. Recent advances in COPD: pathophysiology, respiratory physiology and clinical aspects, including comorbidities. Eur Respir Rev 2009;18:198-212.

3 Sinden NJ, Stockley RA. Proteinase 3 activity in sputum from subjects with alpha-1-antitrypsin deficiency and COPD. Eur Respir $J$ 2013;41:1042-50.

4 Barnes PJ. Inflammatory mechanisms in patients with chronic obstructive pulmonary disease. J Allergy Clin Immunol 2016;138:16-27.

5 Brode SK, Ling SC, Chapman KR. Alpha-1 antitrypsin deficiency: a commonly overlooked cause of lung disease. CMAJ 2012:184:1365-71.

6 McCarthy C, Reeves EP, McElvaney NG. The role of neutrophils in alpha-1 antitrypsin deficiency. Ann Am Thorac Soc 2016;13 Suppl 4:S297-304.

7 Leung SS, Lee P, Most JE, et al. Images in COPD: idiopathic emphysema in a never smoker. Chronic Obstr Pulm Dis 2020;7:130-3.

8 Foreman MG, Campos M, Celedón JC. Genes and chronic obstructive pulmonary disease. Med Clin North Am 2012;96:699-711.
9 Stockley RA, Edgar RG, Pillai A, et al. Individualized lung function trends in alpha-1-antitrypsin deficiency: a need for patience in order to provide patient centered management? Int J Chron Obstruct Pulmon Dis 2016;11:1745-56.

10 Demkow U, van Overveld FJ. Role of elastases in the pathogenesis of chronic obstructive pulmonary disease: implications for treatment. Eur J Med Res 2010;15 Suppl 2:27-35.

11 Rønnow SR, Langholm LL, Sand JMB, et al. Specific elastin degradation products are associated with poor outcome in the eclipse COPD cohort. Sci Rep 2019;9:4064.

12 Thulborn SJ, Mistry V, Brightling CE, et al. Neutrophil elastase as a biomarker for bacterial infection in COPD. Respir Res 2019;20:170.

13 Sand JMB, Knox AJ, Lange P, et al. Accelerated extracellular matrix turnover during exacerbations of COPD. Respir Res 2015;16:69.

14 Fermont JM, Masconi KL, Jensen MT, et al. Biomarkers and clinical outcomes in COPD: a systematic review and meta-analysis. Thorax 2019;74:439-46.

15 Rabinovich RA, Miller BE, Wrobel K, et al. Circulating desmosine levels do not predict emphysema progression but are associated with cardiovascular risk and mortality in COPD. Eur Respir J 2016;47:1365-73.

16 Sidhaye VK, Nishida K, Martinez FJ. Precision medicine in COPD: where are we and where do we need to go? Eur Respir Rev 2018;27:180022.

17 Cazzola M, Calzetta L, Rogliani P, et al. The challenges of precision medicine in COPD. Mol Diagn Ther 2017;21:345-55.

18 Woodruff PG, Agusti A, Roche N, et al. Current concepts in targeting chronic obstructive pulmonary disease pharmacotherapy: making progress towards personalised management. Lancet 2015;385:1789-98.

19 Chapman KR, Burdon JGW, Piitulainen E, et al. Intravenous augmentation treatment and lung density in severe $\alpha 1$ antitrypsin deficiency (RAPID): a randomised, double-blind, placebo-controlled trial. Lancet 2015;386:360-8.

$20 \mathrm{Ma} \mathrm{S}$, Lin YY, Cantor JO, et al. The effect of alpha-1 proteinase inhibitor on biomarkers of elastin degradation in alpha-1 antitrypsin deficiency: an analysis of the RAPID/RAPID extension trials. Chronic Obstr Pulm Dis 2016;4:34-44.

21 Coxson HO, Dirksen A, Edwards LD, et al. The presence and progression of emphysema in COPD as determined by CT scanning and biomarker expression: a prospective analysis from the ECLIPSE study. Lancet Respir Med 2013;1:129-36.

22 Pompe E, Strand M, van Rikxoort EM, et al. Five-year progression of emphysema and air trapping at CT in smokers with and those without chronic obstructive pulmonary disease: results from the COPDGene study. Radiology 2020;295:218-26.

23 Schroeder JD, McKenzie AS, Zach JA, et al. Relationships between airflow obstruction and quantitative CT measurements of emphysema, air trapping, and airways in subjects with and without chronic obstructive pulmonary disease. AJR Am J Roentgenol 2013;201:W460-70.

24 Stockley RA, Parr DG, Piitulainen E, et al. Therapeutic efficacy of $\alpha-1$ antitrypsin augmentation therapy on the loss of lung tissue: an integrated analysis of 2 randomised clinical trials using computed tomography densitometry. Respir Res 2010;11:136.

25 Global Initiative for Chronic Obstructive Lung Disease. Global strategy for the diagnosis, management and prevention of COPD: global initiative for chronic obstructive lung disease (2015 report), 2015. Available: http://goldcopd.org/ [Accessed 03 Aug 2015].

26 Sieren JP, Newell JD, Judy PF, et al. Reference standard and statistical model for intersite and temporal comparisons of CT attenuation in a multicenter quantitative lung study. Med Phys 2012;39:5757-67.

27 European Federation of Pharmaceutical Industries and Associations. The EFPIA code of practice, 2019. Available: https://www.efpia.eu/ relationships-code/the-efpia-code/ [Accessed 01 Mar 2020].

28 International Federation of Pharmaceutical Manufacturers \& Associations. IFPMA code of practice, 2019. Available: https://www. ifpma.org/resource-centre/ifpma-code-of-practice-2019/ [Accessed 01 Mar 2020].

29 Pharmaceutical Research and Manufacturers of America. PhRMA code on interactions with health care professionals, 2019. Available: https://www.phrma.org/-/media/Project/PhRMA/PhRMA-Org/ PhRMA-Org/PDF/A-C/Code-of-Interaction_FINAL21.pdf [Accessed 01 Mar 2020].

30 Macintyre N, Crapo RO, Viegi G, et al. Standardisation of the singlebreath determination of carbon monoxide uptake in the lung. Eur Respir J 2005;26:720-35.

31 ATS Committee on Proficiency Standards for Clinical Pulmonary Function Laboratories. ATS statement: guidelines for the six-minute walk test. Am J Respir Crit Care Med 2002;166:111-7. 
$32 \mathrm{Koo} \mathrm{H-K}$, Jin KN, Kim DK, et al. Association of incidental emphysema with annual lung function decline and future development of airflow limitation. Int J Chron Obstruct Pulmon Dis 2016;11:161-6.

33 Nishimura M, Makita $\mathrm{H}$, Nagai K, et al. Annual change in pulmonary function and clinical phenotype in chronic obstructive pulmonary disease. Am J Respir Crit Care Med 2012;185:44-52.

34 Welte T, Vogelmeier C, Papi A. COPD: early diagnosis and treatment to slow disease progression. Int J Clin Pract 2015;69:336-49.

35 Tantucci C, Modina D. Lung function decline in COPD. Int J Chron Obstruct Pulmon Dis 2012;7:95-9.

36 Nishimura K, Izumi T, Tsukino M, et al. Dyspnea is a better predictor of 5-year survival than airway obstruction in patients with COPD. Chest 2002;121:1434-40.

37 Gans MD, Gavrilova T. Understanding the immunology of asthma: pathophysiology, biomarkers, and treatments for asthma endotypes. Paediatr Respir Rev 2020;36:118-27.

38 Zemans RL, Jacobson S, Keene J, et al. Multiple biomarkers predict disease severity, progression and mortality in COPD. Respir Res 2017;18:117.

39 Dickens JA, Miller BE, Edwards LD, et al. COPD association and repeatability of blood biomarkers in the ECLIPSE cohort. Respir Res $2011 ; 12: 146$

40 Crossley D, Renton M, Khan M, et al. CT densitometry in emphysema: a systematic review of its clinical utility. Int J Chron Obstruct Pulmon Dis 2018;13:547-63.

41 Lowe KE, Regan EA, Anzueto A, et al. COPDGene ${ }^{\circledR}$ 2019: redefining the diagnosis of chronic obstructive pulmonary disease. Chronic Obstr Pulm Dis 2019;6:384-99.

42 Ritchie Al, Donaldson GC, El-Emir E. The British Lung Foundation Early COPD Consortium - recruitment and results from an initia pilot. Am J Respir Crit Care Med 2019;199:A3280 https://www. atsjournals.org/doi/abs/10.1164/ajrccm-conference.2019.199.1_ MeetingAbstracts.A3280

43 Vestbo J, Anderson W, Coxson $\mathrm{HO}$, et al. Evaluation of COPD Longitudinally to Identify Predictive Surrogate End-points (ECLIPSE). Eur Respir J 2008;31:869-73.
44 Couper D, LaVange LM, Han M, et al. Design of the Subpopulations and Intermediate Outcomes in COPD Study (SPIROMICS). Thorax 2014;69:492-5

45 Karch A, Vogelmeier C, Welte T, et al. The German COPD cohort COSYCONET: aims, methods and descriptive analysis of the study population at baseline. Respir Med 2016;114:27-37.

46 Bourbeau J, Tan WC, Benedetti A, et al. Canadian Cohort Obstructive Lung Disease (CanCOLD): fulfilling the need for longitudinal observational studies in COPD. COPD 2014;11:125-32.

47 Regan EA, Hokanson JE, Murphy JR, et al. Genetic epidemiology of COPD (COPDGene) study design. COPD 2010;7:32-43.

48 ClinicalTrials.gov. The BLF early COPD development partnership grant, 2018. Available: https://clinicaltrials.gov/ct2/show/ NCT03480347 [Accessed 18 Feb 2020].

49 Takei N, Suzuki M, Makita H, et al. Serum alpha-1 antitrypsin levels and the clinical course of chronic obstructive pulmonary disease. Int J Chron Obstruct Pulmon Dis 2019;14:2885-93.

50 COPDGene. Study protocol: genetic epidemiology of chronic obstructive pulmonary disease (COPDGene ()$), 2009$.

51 Hoffman EA, Simon BA, McLennan G. State of the art. A structural and functional assessment of the lung via multidetector-row computed tomography: phenotyping chronic obstructive pulmonary disease. Proc Am Thorac Soc 2006;3:519-32 https://www. atsjournals.org/doi/full/10.1513/pats.200603-086MS

52 Ashraf H, Lo P, Shaker SB, et al. Short-term effect of changes in smoking behaviour on emphysema quantification by CT. Thorax 2011;66:55-60.

53 Shaker SB, Stavngaard T, Laursen LC, et al. Rapid fall in lung density following smoking cessation in COPD. COPD 2011;8:2-7.

54 Wan ES, Castaldi PJ, Cho MH, et al. Epidemiology, genetics, and subtyping of preserved ratio impaired spirometry (PRISm) in COPDGene. Respir Res 2014;15:89.

55 Wan ES, Fortis S, Regan EA, et al. Longitudinal phenotypes and mortality in preserved ratio impaired spirometry in the COPDGene study. Am J Respir Crit Care Med 2018;198:1397-405. 